\title{
STUDY ON STOCHASTIC FLOW IN LOW PERMEABILITY SANDSTONE OIL RESERVOIR
}

\author{
Zhaopeng Zhang $^{1}$, Lili Liu ${ }^{2}$ \\ ${ }^{1}$ School of Petroleum Engineering, China University of Petroleum (Beijing), Beijing, China \\ ${ }^{2}$ School of Civil Engineering and Architecture, Southwest Petroleum University, Chengdu, China
}

\begin{abstract}
Reservoir porous medium is random, in terms of oil and gas for low permeability reservoirs. Consider the random nature of the reservoir will enable to improve the accuracy of numerical reservoir simulation. Based on extensive research, for the stochastic nature of the reservoir from the two aspects of physical simulation, numerical simulation, the relevant international studies were reviewed. The research trends of stochastic flow in low permeability oil reservoir were pointed out at last.
\end{abstract}

Keywords: Stochastic Flow; Low Permeability Reservoir, Numerical Simulation, Physical Modeling

\section{INTRODUCTION}

Energy is the foundation of modern economic and social development is an important limitation on the economic and social development, energy security is related to economic security and national security. In our current energy mix, oil still occupies a pivotal position in the primary energy to keep oil production and an increase in long-term stability, to ensure energy security is crucial. To do this, one needs to have a reasonable exploitation of proven reserves, increase its recovery rate; on the other hand, also requires active exploration of new oil and gas resources. However, in recent years, oil and gas exploration and practice has shown that in the new proven reserves of crude oil in low permeability reservoir reserves accounting for about two-thirds of reserves; in 2008, new proven oil reserves of China submitted a low permeability oil Tibetan proportion of even up to $82 \%$. Low permeability reservoirs have low permeability, low reserves abundance, poor reservoir characteristics and development is difficult, it has become the main factors restricting China's oil and gas resources to achieve sustainable development. Whether the existing oil and gas resources recovery rate, or low permeability oil and gas resources exploitation of new proven efficient, they are related to the complex geology of oil and gas exploration and exploitation problem, therefore, national long-term
Science and Technology Development Plan (2006 - 2020) explicitly to the complex geology of oil and gas exploration development and utilization of energy issues into key areas of priority themes.

Energy is the foundation of modern economic and social development is an important limitation on the economic and social development, energy security is related to economic security and national security. In our current energy mix, oil still occupies a pivotal position in the primary energy to keep oil production and an increase in long-term stability, to ensure energy security is crucial. To do this, one needs to have a reasonable exploitation of proven reserves, increase its recovery rate; on the other hand, also requires active exploration of new oil and gas resources. However, in recent years, oil and gas exploration and practice has shown that in the new proven reserves of crude oil in low permeability reservoir reserves accounting for about two-thirds of reserves; in 2008, new proven oil reserves of China submitted a low permeability oil Tibetan proportion of even up to $82 \%$. Low permeability reservoirs have low permeability, low reserves abundance, poor reservoir characteristics and development is difficult, and it has become the main factors restricting China's oil and gas resources to achieve sustainable development. Whether the existing oil and gas resources recovery rate, or low 
permeability oil and gas resources exploitation of new proven efficient, they are related to the complex geology of oil and gas exploration and exploitation problem, therefore, national long-term Science and Technology Development Plan (2006 - 2020) explicitly to the complex geology of oil and gas exploration development and utilization of energy issues into key areas of priority themes.

Fluid mechanics is an important branch of fluid mechanics is the study of fluid flow in porous media disciplines. The process of oil and gas but also oil and gas development process of this special fluid in the reservoir this particular porous media flow, therefore, with the seepage mechanics studied by means of the flow of oil and gas in the reservoir is complex geology of oil and gas exploration and exploitation in important One issue. In order to study the flow of fluid within the reservoir, you must first multi-pore structure within the reservoir rock is described in line with the actual situation, for people depending on the nature and actual existing computing capacity of the porous medium, the porous medium pore structure can be simplified and assumptions made many microscopic physical models. Among them, there are Bernoullis slit model, Poisseuille single-tube model, Wyllie and Gardner et al., Straight capillary tube model, Cornell, Kata et al slanted capillary model and Scheidegger, who bent capillary model, Irmay, Snow, Parsons et al porous media model was constructed with three sets of orthogonal capillary constant width slit and slit group represented. Other microscopic model as well as the hydraulic radius model Kozeny et al., Rumer, Iberall et al flow resistance model, Scherdegger communication channels statistical model, Ferrandon a cylinder, prism combination microscopic structure considerations vector model, Laroche C. et al Run three-dimensional effect of wet microscopic model. These models through the design of each model and the simplified method, the application of different mathematical means of analysis and calculation process of fluid flow in porous media, with their different characteristics. But these simplified models are mostly used to explain the introduction of Darcy's law and so on, in the application has been extremely limited. Considering the randomness of rocks and other porous media deposition process, $\mathrm{Hu}$ Xuetao, who will be randomly theory into microscopic simulation in porous media, using stochastic network simulation study microscopic pores seepage problems. This approach can better characterize the microstructure of the static properties of the porous medium, but still there is a gap between the calculated and experimental values of permeability coefficient, the reason, many believe that the existing methods of reservoir rock porosity characteristics within the media is deterministic, pore characteristics determine flow parameters will inevitably lead to uncertainty; but in fact, a lot of research indicates that, for this type of rock and soil media, internal pore characteristics description parameters often have a lot of randomness and uncertainty, randomness is bound to lead to random pore structure of its mechanical parameters of seepage, therefore, characterized by having a random distribution of variables, and values to describe fluid mechanics parameters of rock and soil media is not a defined more in line with project the actual situation.

\section{RESEARCH STATUS ON PHYSICAL}

\section{MODELLING}

Modern development of non-destructive detection technology and image processing technology provides a powerful technical means for understanding the microstructure of engineering within the pores of the rock media. Joshi was first proposed reconstruction rock pore space of statistics, statistics on which to rock thin section analysis was based on random establish a porosity model and the model will be able to continue to adjust until the model and the real nature of the rock samples obtained with the nature of statistics Until agreement, Quiblier further development of various reconstruction algorithms Joshi raised. Hidajat and other statistical method based on the use of thin build three-dimensional porous media; Liang et statistical results based on the two-dimensional image on a sheet of particle size distribution of rock to build a three-dimensional porous media; Okabe and Blunt according to pore sheet degree and related functions to build a three-dimensional data field. Bakke and Oren combines rock particle size distribution, for the first time by the formation of sedimentary rocks (including deposition, compaction and diagenetic) analog established pore space, and then reconstructed by means of a Voronoi polyhedron of pore network model. Dunsmuir, Vogel and other X-ray tomography technology to build a rock-dimensional image; Hazlett, Coles and other development of nuclear magnetic 
resonance, or a two-dimensional thin slice method of pore space image rendering technique. Vogel and other soil samples were CT scan and the establishment of a three-dimensional pore model; Hidajat and other porous media based on the statistical results to establish a two-dimensional image of the sheet rock in particle size distribution; Coker such as the use of X-ray tomography technology has been rock image reconstruction; Manwart others, based on stochastic modeling methods, the establishment of a three-dimensional porous medium data volume; in pore throat size and aspect extraction of topology information, Jerauld, Salter, Blunt and King and Dixit lattice with cube and cylindrical capillary network model was constructed and the coordination number of network simulation under different conditions; Adler and Thovert studied by the sphere to simulate the rock formation; Lindquist and Hou Jie The porous media 3D data using image processing techniques to obtain the topological structure of the pore space; on the basis of non-destructive detection technology and image processing technology, based on the core X-ray, CT, magnetic resonance imaging and other chromatography data, analysis of reservoir rock the characteristics of the pores, and the use of statistical methods, the random distribution of Pore description of the parameters established in the stochastic model of pore distribution, the uncertainty and randomness with probability or other related values (eg statistical elements) to Characterization will help deepen the understanding within the pores of the rock features, make the model closer to the actual project.

Early fluid mechanics is mainly used in traditional geotechnical engineering groundwater exploitation, Dam, etc. Therefore, for a long time, seepage mechanics study focused on macro research methods, namely the formation or soil samples and rock or soil for the study, work scope and depth of research seepage macro has reached a considerable degree, research in this area is undoubtedly necessary and important. However, macro research alone means a lot of problems, particularly issues related to percolation mechanism method, it is difficult to obtain a better solution. Microscopic studies seepage seepage is an important means understanding the law, the microscopic mechanism restricts seepage flow in macroscopic processes, and contribute to the macroscopic flow deeper understanding of the law. Microscopic studies complement each macro flow mechanics; will greatly facilitate the resolution of relevant issues.

There are two ways seepage microscopic studies that physical modeling and numerical simulation. Physical simulation experiment is microscopy observations membership in rock samples and model fluid flow in detail, the mechanism and law, and by means of video and camera recording. China started earlier in terms of microscopic physics simulation, began this work in 1978, 81 year of publication MICROVADOSE results. Developed in 1985 by the 11 technical support successful simulation and testing technology, can be a good mimic natural pore topology fractured medium pore surface roughness and wettability and complex flows under high temperature and pressure, can also be simulated the impact of some of the mineral composition of clay minerals, etc., can be measured trace each phase of a multiphase fluid flow. The application of this simulation testing technology has been studied systematically in multiphase flow, a non-Newtonian fluid flow, physical and chemical seepage and non-isothermal flow and other aspects, the new found some seepage mechanism and laws. For example, the discovery of the "hole surrounded by big hole" mechanism when water two-phase flow, the original that polymer flooding can only improve sweep efficiency does not improve wash the oil factor, but porosity level experiments showed that the polymer will not only improve sweep efficiency, but also can improve wash the oil factor. The discovery of the polymer injection enhanced oil recovery in the industry provides an important new scientific evidence. The past two decades, research seepage microscopic physics simulation at home and abroad have been more and more attention. In China, a lot of oil research institutes have microcosmic study at this level as a regular means of support and macro experiments carried out. Physical simulation of the microscopic flow, whether it is hardware, or microscopic percolation theory, temporarily in the international advanced level.

\section{RESEARCH STATUS ON NUMERICAL}




\section{SIMULATION}

Microscopic physical simulation has its own shortcomings, such as high cost, time consuming, and other factors that may be considered less. Therefore, the development of porous media flow microscopic simulation study has important significance. At present, domestic and foreign research results related to seepage microscopic simulation is also less, there are mainly lattice gas automata and percolation theory two methods, studies the microscopic flow characteristics minority fractal theory. In terms of lattice gas automata, Rothman such as the use of porous lattice gas model to study the mechanics of the basic problems, Darcy Theorem permeate flow relationship with the pressure gradient. Its basic idea is to simulate flow in a space set up many random obstacles to prevent fluid through, thereby reducing the permeability. Liu said $\mathrm{Wu}$, etc. using the lattice gas (Lattic Gas) method simulate natural rock pores in the two-phase flow, to calculate the relative permeability curves and residual oil saturation, the calculated results and the results compared to the physical simulation, although quantitatively there are relatively large errors, but are qualitatively consistent with the. Zheng Zhong et utilize 13-bit multi-speed regular hexagonal lattice gas automata model, discussed and permeability of fluid flow in porous media lattice gas automata model parameter calculation method, the application of the porous medium geometry of the model computer generated and coke porous medium fluid flow is simulated. $\mathrm{Xu}$ Yousheng, etc. are Lattic Boltzmann model to study the use of porous media water flooding process and the microscopic mechanism. Percolation theory is a way to explain the fluid Broadbent and hammersley in 1957 proposed transport phenomena in disordered media. In the study of porous media flow characteristics in recent years it has attracted people's attention. Wang Jinxun, Liu Qingjie etc. according to percolation theory, combined with the network model to calculate the porosity levels and water relative permeability curves Buckley-Leverett equation based on the theory of continuous media. JP Tian through the structure of the different percolation Group, which was regarded as porous media communication, and that the grid is the pore, the key for the throat, and throat radius obey beta distribution, and then to the center of the injection point, the use of ultrarelaxation techniques, gained percolation Group viscous fingering image. Hu Jiao and other sounds in order to reveal the multiphase fluid flow in porous media and the displacement process mechanism, based on the percolation theory of probability model breakthrough pressure, using the renormalization method to solve a simple cubic pore topology and a certain pore size Critical probability distribution occur under the three-dimensional network "breakthrough" is to determine the function of breakthrough pressure in porous media and porous media porosity, permeability, fluid properties and the critical probability. Donghui such as the use percolation theory to study the process of mass transport in porous media, the law of diffusion bonding rate under different pore channels were analyzed. Numerical simulation of microscopic lattice gas seepage and percolation theory, all means of knowledge of other disciplines to conduct research, contact the hydrodynamics theory is unlikely, and research ideas and methods of hydrodynamics far. As we all know, seepage mechanics is a branch of fluid mechanics discipline, from the general to the particular sense, there is no necessary link between the internal fluid mechanics and fluid mechanics. Based on fluid mechanics and micro flow boundary layer theory, differential equations under consideration microfluidic boundary and pore fluid motion to simulate the movement of fluid within the pores at the micro level; on this basis, to derive the same initial and boundary conditions Under conditions corresponding macroscopic flow parameters, will help obtain more reliable rock macroscopic flow parameters.

When the first study of pore fluid flow problems assume solid skeleton is not deformed, and later many scholars have studied the influence of rock deformation on its permeability, that the permeability of the rock mass deformation is not uniform unchanging constant, it varies pore pressure and confining pressure changes. In this case the law of fluid flow in a deformable rock and deformation of the analysis, beyond the scope of fluid mechanics and rock mechanics, in addition to the need for external flow field equations and rock deformation field or stress field equations, but also on such issues to FSI analysis. Fluid-structure coupling theory in reservoir induced seismicity, dam seepage, open pit slope stability, tunnel gushing water and other geotechnical engineering fields have been widely used. Through a large number of site development practices, experimental and 
theoretical studies show indoor, reservoir development process is a multi-phase fluid flow and reservoir rock deformation dynamic coupling process. Applied research of reservoir fluid-solid coupling theory in gas field development project in quite late, but in recent years has received increasing attention: Finol and consider the establishment of a two-dimensional two-phase flow Farouq solid rock compaction Effect fluid flow dynamics and development coupling model; Xiaoming Ji based on porosity, etc. - differential dual porosity media flow fractured rock solid coupling calculation, was presented with the rock mass stress state associated permeability coefficient formula; $T$ \& T-based dual medium model derived rock mass stress field and the non-linear coupling analysis of seepage field; Yongming $\mathrm{Li}$ holes on the basis of a single permeability reservoir model, considering the natural fracture permeability changes with pressure, the establishment of oil-water double mathematical model of reservoir fracturing medium; with Davydenko consider deformation stress sensitive formations media, the establishment of a new dual stress sensitive formations fractal media mathematical model. Therefore, in the flow microscope simulation, consider the impact of changes in flow stress state parameters (especially flow rate), using fluid - solid coupling methods, analyzing macroscopic flow parameters on the stress sensitivity parameters, thereby establishing core macro-flow parameters functional relationship between stress and the macroscopic flow parameters obtained will be more in line with the late development of gas fields reservoir permeability decreases rule.

Flow, mechanical parameters of rock and soil media often have uncertainty and randomness, when the prediction of groundwater resources based on these parameters, reservoir development effectiveness evaluation, underground contaminant transport control and other engineering problems, bound with uncertain parameters to predict or evaluate the results of the uncertainty, for practical engineering problems, it is often necessary to quantify this uncertainty evaluation or to give confidence interval. Uncertainties in the engineering of quantitative methods, the most common is the numerical simulation of Monte-Carlo, and the Monte-Carlo method and finite element method combined to become a common method for solving engineering problems, the probability of which is based on classical spatial variability Statistics generated by computer simulation parameters large enough sample space to simulate spatial variability of parameters, the parameters in the sample space for each sample point, and analyzed by deterministic finite element method, so that you get a large enough set of solutions on the solution set of statistical analysis, probability and statistics can be obtained characteristic stress, pore pressure, etc. are required. Because it is directly based on a large number of deterministic finite element calculation, when the sample size is large enough, Monte-Carlo finite element method in the study of early random seepage problem is considered to be one of the more reliable and more precise method. Because of Monte-Carlo finite element method to conduct a large number of deterministic finite element calculation, when the need for fluid flow in the medium will be more refined description, it is often because of the need for a large number of intermediate state data, mathematical statistics and inefficient, stochastic finite element method to solve this problem provides a more effective means, the method based on medium stress, seepage control differential equations on the use of certain discrete way to create finite element equation; while using random field theory Spatial variability of medium and medium parameters describe discrete random order for unit parameter assignment. Stochastic finite element prediction of groundwater, oil and gas development and other fields has been widely used: Yajun such as the use of stochastic finite element analysis method to study the three-dimensional Anisotropic Random Seepage Field heterogeneous stable long Jiang Jingnan main dikes random seepage field simulation result of the impact parameter and the random boundary conditions, and sensitivity analysis; Numerical simulation of underground non-uniformity in the unsaturated zone of underground caverns and other seepage problem Li Guomin performs the medium parameters of sensitivity analysis; Yajun such as the use of stochastic finite element analysis method to study the long Jiangjing Nan Main Dike seepage field with random parameters and random boundary conditions on the random flow field impact and stochastic simulation parameters and boundary conditions random random sensitivity; Yang Dong, Zhao rose through the cracks in the gas-liquid two-phase fluid flow analysis of the phenomenon of critical reasoning, described this phenomenon establish mathematical model of random mixing and application of the model, the probability 
distribution of critical percolation phenomenon, critical gas seepage area Distribution of microscopic distribution of water communication groups and critical flow area to simulate the pressure gradient, which reveals the mechanism of this phenomenon; LI Jin-hui, Wang Yuan, the permeability coefficient is introduced as a random expression of functional variation In the formula, use the small parameter perturbation method derived established a three-dimensional steady flow of random variational principle and the corresponding stochastic finite element formulation; Ganis such as the use of stochastic finite element method of fluids in porous media random construction mold, uncertainty state variables were quantitative analysis; Kaminski et fluid state of the fluid flow parameters in the process of first and second order sensitivity has been studied. Therefore, the use of stochastic finite element method, consider the random distribution of flow parameters in the study will be quantitative medium pressure fluid flow parameters of state output sensitivity; also may optionally fluid flow within the pores of a random process, fine Fluid flow processes in random media to deepen understanding of the laws of the seepage.

\section{CONCLUSIONS AND UNDERSTANDING}

Stocktaking research and analysis, future research will focus on random seepage in the following areas: reservoir core based on CT data, the geometric features in the study of pore description of parameters and statistics law, establishing the rock pores and stochastic models pore structure reconstruction cores; study $\mathrm{CT}$ image reconstruction within the pore structure characteristics on the statistical significance of the goodness of fit criterion, inspection and correction model. Consider the interaction between the pore fluid and the solid wall, establishing Differential pore fluid movement, the preparation simulation software to simulate the movement of fluid within the pores at the micro level and the micro CT binding displacement experiment tested; and then derived and The corresponding macroscopic flow parameters and flow parameters checked against experimentally obtained core flooding. Macroscopic flow parameters obtained and random pore model established based on using the movement of stochastic finite element method core pore fluids under different stress state, thereby establishing core macroscopic flow parameters and fluid physical parameters, core mechanics parameters and cores functional relationship between stress state. Carry out project implementation will be transformed to obtain macroscopic flow parameters for numerical simulation of micro issues, in order to obtain macroscopic flow parameters to provide a more economical new way; results can be used to adjust the gas field development plan, the remaining oil distribution forecast three developed areas, such as mining program.

\section{REFERENCES}

[1] Laroche C.. Network modeling to predict the effect of wettability heterogeneities on multiphase flow[J]. Journal of Society of Petroleum Engineer. 1999, 39(2):113-123

[2] Satterfield S.N. Mass Transfer in Heterogeneous Catalysis. Massachusetts: MIT Press, 1990:33-40

[3] Dullien F. A. L. Porous Media: Fluid Transport and Structure. NewYork: Academic Press Inc., 1979:223-230

[4] Liu Jianjun, Lin Lijun, Song Rui, Zhao Jinzhou. A Pore Scale Modelling of Fluid Flow in Porous medium Based on Navier-Stokes Equation. Disaster Advances, 2013, 6(S1): 129-136

[5] Xin F. Study on Structure and Gas Diffusion in Porous Media with Percolation Network Model [Dissertation]. Tianjin: Tianjin University, 1996

[6] Mc Dougall S R, Sorbie K S. The Combined Effect of Capillary and Viscous Forces on Waterflood Displacement Efficiency in Finely Laminated Porous Media, SPE26659, 1993

[7] Wilkinson D, Willemsen J F. Invasion Percolation: A New Form of Percolation Theory. J. Phys. A, 1983, 16:3365-3376

[8] Mohanty K. Physics of Oil Entrapment in Water-wet Rock. SPE Reservoir Engineering. 1987,(2 ): 113-125

[9] Richard Durrett. Oriented Percolation in Two Dimensions. The Annuls Probability, 1994,12(4):999-1040

[10] Morrow N R. Interfacial Phenomena in Petroleum Recovery. Marcel Dekker, Inc. 1991

[11] Jadhunandan PP, et al. Effect of Wettability on Waterflood Recovery for Crude-oil/Brine/Rock 
Systems. SPE Reservoir Engineering, 1995, (2 ):40

[12] Blunt M J, King M J. Simulation and Theory of in Porous Media. Phys RevA, 2002,46(12):1345-1359

[13] Anderson W. Wettability Literature Survey-Part I: Rock/Oil/Brine Interactions and the Effects of Core Handling on Wettability.PT, 1986, (10):1125-1145

[14] Barrufet M.A. R J White. A Waterflood Model Based upon Percolation Theory Concepts. SPE27018, 1994

[15] Liu Jianjun, Song Rui. Numerical Simulation Study on Non-Darcy Seepage Mechanism in micro-pore based on the Micro-fluidic Boundary Layer Theory. Research Journal of Chemistry and Environment, 2014, 18(5):1-6

[16] Treiber L E, Archer D L. A Laboratory Evaluation of the Wettability of Fifty Oil Producing Reservoir. Soc. Petro. Eng. J. 1972,12(6): 531

[17] Lenormand R. Two-phase Flow Experiments in a two-dimensional permeable medium. Phys. Chem. Hydrodyn. 1995,(6):497-506

[18] Larson R G. Displacement of Residual Nowetting Fluid from Porous Media. J. Chem. Eng. Sci., 1991,36:75-85

[19] Richard Durrett. Oriented Percolation in two dimensions. The Annuls Probability, 1999,12(4):999-1040

[20] M . Joshi . Ph . D . thesis,Univ . of Kansas,Lawrence, 1974.

[21] Quiblier,J . A New Three-dimensional Modeling Technique for Studying Porous Media. J. Colloid Interface Sci. ,1984; 98: 84-102.

[22] Hidajat,I • ,A • Rastogi,M . Singh and K. K. Mohanty. Transport Properties of Porous Media from Thin-sections . SPE 69623,SPE LACPEC. March 25-27,2001.

[23] Liang,Z. R. ,P. C. Phillippi,C. P. Fernandes,and F. S. Magnani. Prediction of Permeability from the Skeleton of Three-dimensional Pore Structure. SPE Reservoir Evaluation and Engineering,1999; 2(2): 161-168.

[24] Okabe,H. and Blunt,M. J. Multiple-point Statistics to Generate Geologically Realistic Pore-scale Representations. Proceedings of the Society of Core Analysts Annual Meeting,2003.

[25] Bakke,S. , and P. E. Oren. 3-D Pore-scale Modelling of Sandstones and Flow Simulations in the Pore
Networks. SPE J. ,1997; 2: 136-149.

[26] Dunsmuir,J. H. ,S. R. Ferguson,K. L. D'Amico, and J. P. Stokes. X-ray Microtomography. A New Tool for the Characterization of Porous Media . Proceedings of the 1991 SPE Annual Technical Conference and Exhibition . SPE 22860,Soc. Of Petroleum Engineers,Dallas, 1991.

[27] Vogel,H. J. , and K. Roth. Quantitative Morphology and Network Representation of Soil Pore Structure. Advances in Water Resources,2001; 24: 233-242.

[28] Hazlett,R. D. Simulation of Capillary-dominated Displacements in Micro-Tomographic Images of Reservoir Rocks. Transport in Porous Media,1995; 20: 21-35.

[29] Coles,M. E. ,R. D. Hazlett,P. Spanne,et al. Pore Level Imaging of Fluid Transport Using Synchrotron X-ray Microtomography . Journal of Petroleum Science and Engineering,1998; 19: 55-63.

[30] Vogel H J,Roth K.A new approach for determining effective soil hydraulic functions.Eur $\mathrm{J}$ Soil Sci, 1997,49(4):547-556

[31] Hidajat I,Rastogi A,Singh M,et al.Transport properties of porous media from thin-sections.SPE J,2002,7(1):40-48

[32] Coker D A,Torquato S,Dunsmuir J H.Morphology and physical properties of Fontainebleau sandstone via a tomographic analysis.J Geophys Res-Solid Earth,1996,101:17497-17506

[33] Manwart C,Torquato S,Hilfer R.Stochastic reconstruction of sandstones.Phys Rev E,2000,62:893-899

[34] Lindquist W B,Venkatarangan A B.Investigating 3D geometry of porous media from high resolution images.Phys Chem Earth Part A-Solid Earth Geod,1999,24(7):593-599

[35] Hou J,Zhang S K,Sun R Y,et al.Reconstruction of 3D network model through CT scanning.In:Proceedings of the SPE Europec/EAGE Annual Conference and Exhibition. Richardson: Society of Petroleum Engineers, 2007.1-10

[36] Liu Jianjun, Song Rui. Improvement of predictions of petrophysical transport behavior using three-dimensional finite volume element model with 
micro-CT images. Journal of Hydrodynamics. 2015, 27(2): 234-241

[37] Yan Q.L. Micro-Visualization Study of Fluid Flow Behaviors in Porous Media. Computer Methods and Advances in Geomechanics. 1994,(2):88-92

[38] Liu Jianjun, Li Quanshu. Numerical Simulation of Injection Water Flow through Mudstone Interlayer in Low Permeability Oil Reservoir Development. Disaster Advances, 2012, 5(4): 1639-1645

[39] Read,P. A. and Ringen, J. K. The Use of laboratory Tests to Evaluate Scaling Problems during Water Injection. SPE10593,1992

[40] S.S.Augustithis. On the Micro and Macro Scale Phenomena. Earth Sciences Frontier,1995, 2(1-2):26-28

[41] Song Rui, Liu Jianjun, Qin Dahui. Numerical Simulation of Two Phase Flow in Reconstructed Pore Network Based on Lattice Boltzmann Method. International Journal of Computer Science Issues, 2013, 10(1):193-200

[42] Rothmanm, D.H. Cellular-automaton fluids: a model for flow in porous media, preprint. MIT Earth Research Lab. (1988)

[43] Balaubramanian, K., Hayot, and Seam,W.F. Darcy's law from lattice gas hydrodynamics. Preprint. Ohio State University, 1987

[44] Muskat M. The Flow of Homogeneous Fluids through Porous Media[M], New York: McGrawHill Press, 1946

[45] Jianjun Liu, Rui Song, and Mengmeng Cui, Numerical Simulation on Hydromechanical Coupling in Porous Media Adopting Three-Dimensional Pore-Scale Model. The Scientific World Journal, vol. 2014, Article ID 140206, 8 pages, 2014. doi:10.1155/2014/140206

[46] Larry S.-K. Fung. A coupled geomechanic-multiphase flow model for analysis of in situ recovery in cohensionless oil sand. JCPT, 1992,31(6)56-60.

[47] Ganis, Benjamin. Stochastic collocation and mixed finite elements for flow in porous media. Computer Methods in Applied Mechanics and Engineering, 2008, 197(43-44):3547-3559.

[48] Liu Jianjun, Song Rui, Zhao Jinzhou. Numerical Simulation Research on Seepage Mechanism in
Pore-scale Deformable Porous Media. Disaster Advances, 2013, 6(S1): 49-58

[49] Kaminski, Marcin; Carey, Graham F. Stochastic perturbation-based finite element approach to fluid flow problems. International Journal of Numerical Methods for Heat and Fluid Flow, 2005, 15(7): 671-697.

[50] Liu Jianjun, Liu Xiangui, Ji Youjun, Song Rui. Investigation of Fluid Flow in Fractured Low Permeability Oil Reservoir Coupled with Geostress. Sydney: ST PLUM BLOSSOM PRESS, 2014.3

[51] Junwei Zhang, Yi Zeng, Cheng Lin Zhao. Coupling Analysis of Stress and Seepage of Vertical well's Horizontal Perforation based on GeoStudio. Electronic Journal of Geotechnical Engineering, 2014, 19(F) :1341-1351

[52] Zhu Dapeng, Qin Liangkai, Zuo, Lei. The long-term stability of landslide on condition of fluctuation of reservoir water level in the Three Gorges reservoir area, Electronic Journal of Geotechnical Engineering, 2014, 19 (O):3529-3539

[53] Ji You-Jun, Liu Jian-jun, Cheng Lin-song, Lian Pei-qing. Numerical computation of pressure on the thermal recovery well based on thermal-hydro-mechanical coupling. Engineering Mechanics, 2011, 28(3): 224-229 